\title{
Influence of the reduction temperature on the structure of the metal particles and the metal-support interface of $\mathrm{Pt} / \gamma-\mathrm{Al}_{2} \mathrm{O}_{3}$ catalysts
}

\author{
D.C. Koningsberger ${ }^{\mathrm{a}, *}$, M. Vaarkamp ${ }^{\mathrm{b}}$ \\ ${ }^{a}$ Debye Institute, Utrecht University, P.O. Box 80083, 3508 TB Utrecht, The Netherlands \\ ${ }^{\mathrm{b}}$ Schuit Institute of Catalysis, Eindhoven University of Technology, P.O. Box 513,5600 MB Eindhoven, The Netherlands
}

\begin{abstract}
The structure of the metal particles and the metal-support interface of a $\mathrm{Pt} / \gamma-\mathrm{Al}_{2} \mathrm{O}_{3}$ catalyst was determined by EXAFS after low temperature reduction (LTR: $300^{\circ} \mathrm{C}$ ) and high temperature reduction (HTR: $450^{\circ} \mathrm{C}$ ). The clusters have excellent thermal stability as the particle size remains 12 atoms per cluster upon increasing the reduction temperature from $300^{\circ} \mathrm{C}$ to $450^{\circ} \mathrm{C}$. However, the structure of the metal-support interface is a strong function of the reduction temperature. After LTR and in the presence of chemisorbed hydrogen the metal particles are at a distance of $2.68 \AA$ from the support oxygen ions. This long Pt-O distance is due to the presence of hydrogen between the platinum atoms and the support. Increasing the reduction temperature results in the removal of hydrogen from the metal-support interface, simultaneously placing the metal particles in direct contact with the support oxygen atoms at a distance of $2.28 \AA$. The release of interfacial hydrogen during high temperature reduction is changing the metal-support interaction, which in turn changes the electron density distribution in the metal particles. The electronic and thereby the catalytic properties of the platinum metal particles are therefore a function of the reduction temperature. The results show that the influence of the reduction temperature on the structure of the metal-support interface of platinum particles on a amorphous support is similar to platinum particles which reside in cavities of zeolites.
\end{abstract}

\section{Introduction}

Several studies [1-6] of platinum supported on nonreducible oxides have reported changes in the catalytic performance and the hydrogen chemisorption capacity after high temperature reduction (HTR); however, each has attributed these changes to a different cause. Den Otter and Dautzenberg [1] assumed that the loss in activity was the result of the formation of a $\mathrm{Pt}-\mathrm{Al}$ alloy. Menon and Froment [2] assigned the loss in activity after HTR to the presence of strongly chemisorbed

*Corresponding author. hydrogen. Kramer and Andre [3] proposed support hydrogen adjacent to the platinum particles were responsible for the changes in the catalytic properties. Additionally, Abasov et al. [6] reported that the hydrogen chemisorption capacity of $\mathrm{Pt} / \gamma-\mathrm{Al}_{2} \mathrm{O}_{3}$ decreased upon hydrogen treatment at temperatures above $500^{\circ} \mathrm{C}$. Similarly, decreases in hydrogenolysis activity and hydrogen chemisorption capacity have been reported for platinum in acidic and neutral LTL zeolite $[7,8]$. In these studies the structural characteristics of the catalysts were determined by EXAFS and $\mathrm{H}_{2}$ TPD. It was concluded that after LTR a layer of atomic hydrogen residues between the platinum particle and the zeolite framework. During 
HTR, the interfacial hydrogen is desorbed and platinum is in direct contact with the oxide ions of the support.

In this study, the structure of the metal particles and the metal-support interface of $\mathrm{Pt} / \gamma-\mathrm{Al}_{2} \mathrm{O}_{3}$ has been determined by EXAFS after reduction at $300^{\circ} \mathrm{C}(\mathrm{LTR})$ and $450^{\circ} \mathrm{C}$ (HTR). From the EXAFS results reported here, it is inferred that the influence of the reduction temperature on the chemical and catalytic properties of platinum particles on amorphous supports is similar to the influence on platinum particles dispersed in zeolites.

\section{Experimental}

\subsection{Catalyst preparation}

A $1.0 \mathrm{wt} \% \mathrm{Pt} / \gamma-\mathrm{Al}_{2} \mathrm{O}_{3}$ catalyst was prepared by pore volume impregnation of Ketjen CK-300 $\left(200 \mathrm{~m}^{2} / \mathrm{g}\right.$, $0.6 \mathrm{~cm}^{3} / \mathrm{g}$ ) with an aqueous solution of hexachloroplatinic acid $\left(\mathrm{H}_{2} \mathrm{PtCl}_{6}\right)$. The catalyst was dried in air overnight at $120^{\circ} \mathrm{C}$ before it was reduced at $450^{\circ} \mathrm{C}$ (heating rate $3^{\circ} \mathrm{C} / \mathrm{min}$ ) for $4 \mathrm{~h}$. After reduction the catalyst was flushed with $\mathrm{N}_{2}$ and exposed to air at room temperature

\subsection{EXAFS data collection}

EXAFS spectra were measured at the Synchrotron Radiation Source (SRS) in Daresbury, UK, Wiggler Station 9.2, using a $\mathrm{Si}(220)$ double crystal monochromator. The storage ring was operated with an electron energy of $2 \mathrm{GeV}$ and a current between 120 and $250 \mathrm{~mA}$. At the $\mathrm{Pt}$ $\mathrm{L}_{\text {III }}$ edge $(11564 \mathrm{eV})$, the estimated resolution was $3 \mathrm{eV}$. The monochromator was detuned to $50 \%$ intensity to avoid the effects of higher harmonics present in the X-ray beam. The measurements were done in the transmission mode. To decrease low- and high-frequency noise as much as possible, each data point was counted for $1 \mathrm{~s}$ and six scans were averaged.

The sample was pressed into a self-supporting wafer (calculated to have an absorbance of 2.5) and placed in an in situ cell, with the sample handled in the absence of air. After drying in flowing helium at $120^{\circ} \mathrm{C}$ for two hours the sample was heated at a rate of $5^{\circ} \mathrm{C} / \mathrm{min}$ to the reduction temperature of $300^{\circ} \mathrm{C}$ (sample designated as $\mathrm{R} 300$ ) and $450^{\circ} \mathrm{C}$ (sample designated as R450) in flowing, purified, and dried hydrogen at atmospheric pressure. The sample was cooled to RT under flowing hydrogen and measured at liquid $\mathrm{N}_{2}$ temperature.

\subsection{EXAFS data analysis}

Standard procedures were used to extract the EXAFS data from the measured absorption spectra [8]. The
EXAFS data analysis was performed on an isolated part of the data obtained by an inverse Fourier transformation over a selected range in $r$-space. The isolated final EXAFS functions was obtained by averaging the inverse Fourier transformations of each EXAFS data set. The standard deviations calculated from the data points of the isolated EXAFS functions provided a measure of the random error in the average isolated EXAFS function.

Data for the phase shifts and backscattering amplitudes were extracted from EXAFS data obtained from reference compounds. Pt foil was used as a reference for the $\mathrm{Pt}-\mathrm{Pt}$ contributions and $\mathrm{Na}_{2} \mathrm{Pt}(\mathrm{OH})_{6}$ for the $\mathrm{Pt}-\mathrm{O}$ contributions [8].

The parameters characterizing the high- $Z(\mathrm{Pt})$ and low- $Z(\mathrm{O})$ contributions were determined by multipleshell fitting in $k$-space with optimization in $r$-space. Different backscatterers were identified by applying the difference file technique using phase- and amplitude-corrected Fourier transforms. The optimal combination of the coordination number and the Debye-Waller factor was determined by optimizing the $k^{1}$ and $k^{3}$ weighted Fourier transforms [9]. The errors in the structural parameters were calculated from the covariance matrix, taking into account the statistical noise obtained for the Fourier filtered EXAFS function and the correlations between the refined parameters. The values of the goodness of fit $\left(e_{v}^{2}\right)$ were calculated as outlined in the Report on Standards and Criteria in XAFS Spectroscopy [10].

\section{Results}

The average EXAFS spectra of the six data sets measured after reduction of the catalyst at $300^{\circ} \mathrm{C}$ and $450^{\circ} \mathrm{C}$ are presented in Fig. 1, together with their corresponding Fourier transforms. These figures show that there are changes in the structure of this catalyst upon different reduction treatments. The main $\mathrm{Pt}-\mathrm{Pt}$ peak in the Fourier transform for the sample R450 exhibits a significant shift and higher amplitude at shorter distance. Also the imaginary parts are different between $1.5<R<$ $2.5 \AA$. Fig. 2 displays the normalized $k^{1}$ weighted Fourier transform (Pt-Pt phase and amplitude corrected) for the EXAFS data of sample R300 and for Pt foil. Differences in both the magnitude and the imaginary part of the Fourier transform between $1 \AA<R<3 \AA$ is a clear indication of the presence of additional (low $Z$ ) scatterers besides Pt. Multiple shell fitting $\left(k^{1}, \Delta k=3.5-13.0 \AA^{-1}\right)$ of the Fourier filtered EXAFS $\left(k^{2}, \Delta k=2.7-13.8 \AA^{-1}\right.$, $\Delta R=1.2-3.3 \AA$ ) (denoted by isolated EXAFS) of sample R300 (see Fig. 3) resulted in the identification of two significant contributions (Table 1): a Pt-Pt contribution at $2.76 \AA$ and a $\mathrm{Pt}-\mathrm{O}$ contribution from the metal-support interface at $2.68 \AA$. The goodness of fit $\varepsilon_{v}^{2}$ was 

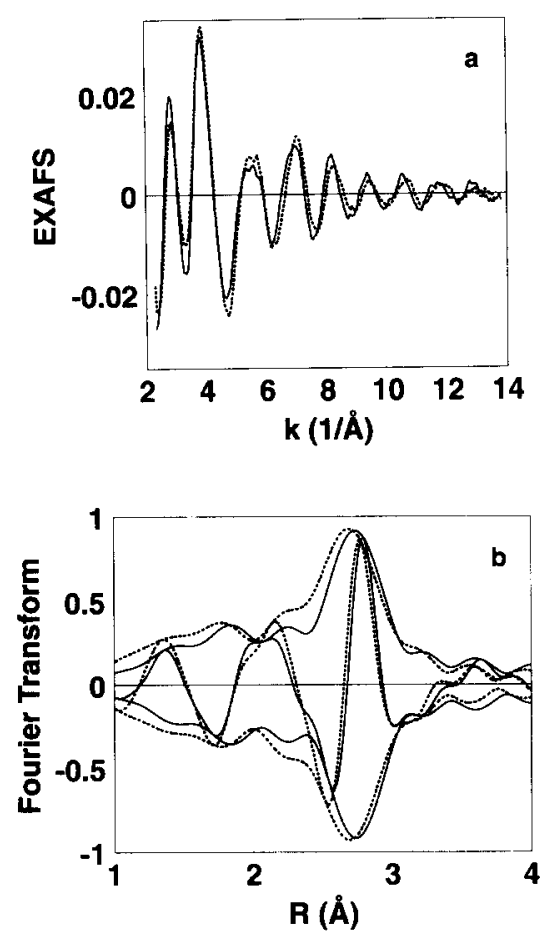

Fig. 1. (a) Raw EXAFS spectra of sample reduced at $300^{\circ} \mathrm{C}$ (solid line) and $450^{\circ} \mathrm{C}$ (dotted line). (b) Fourier transform $\left(k^{1}\right.$, $\Delta k: 3.1-13.1 \AA^{-1}, \mathrm{Pt}-\mathrm{Pt}$ phase and amplitude corrected) of spectra shown in (a).

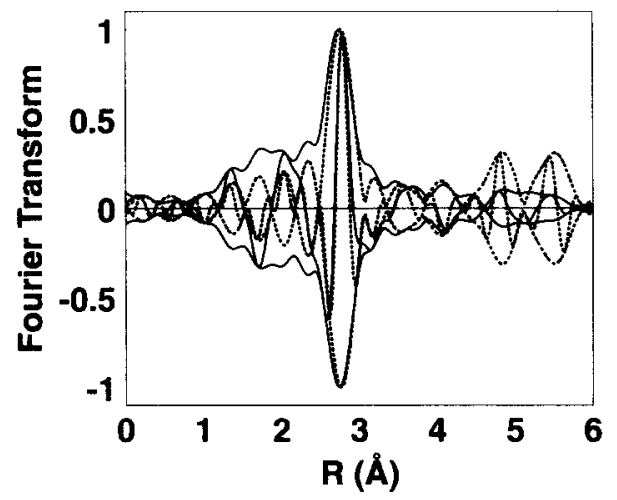

Fig. 2. Normalized Fourier Transform $\left(k^{1}, \Delta k=3.1-13.1 \AA^{-1}\right.$, $\mathrm{Pt}-\mathrm{Pt}$ phase and amplitude corrected) of sample R300 (solid line) and $\mathrm{Pt}$ foil (dotted line).

calculated to be 17.2. The difference file (isolated EXAFS minus calculated Pt-Pt contribution) represents the contribution originating from the metal-support interface of the sample R300 (solid line in Fig. 4(a)). The results of multiple shell fitting $\left(k^{1}, \Delta k=3.5-13.0 \AA^{-1}\right)$ of the

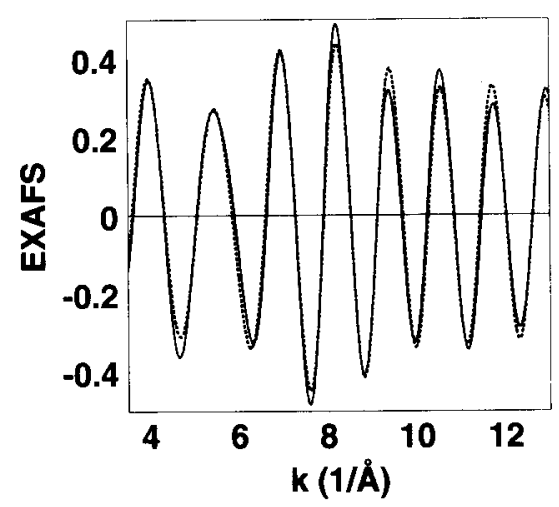

Fig. 3. Fit in $k$-space $\left(k^{2}\right)$ of sample R300. Isolated EXAFS (solid line) and fit (dotted line).

Fourier filtered EXAFS $\left(k^{2}, \quad \Delta k=2.7-12.6 \AA^{-1}\right.$, $\Delta R=1.4-3.3 \AA$ ) for sample $R 450$ are given in Table 1 . The value of the goodness of fit was 6.2. The difference file displayed in Fig. 4(b) contains the contribution from the metal-support interface of sample R450. It is clear that the two contributions are different with a much lower $\mathrm{Pt}-\mathrm{O}$ distance (2.28 $\AA$ ) for the sample $\mathrm{R} 450$.

\section{Discussion and conclusions}

The $\mathbf{P t}-\mathbf{P t}$ first shell coordination number of 5 corresponds to an average platinum particle of 12 atoms. The excellent resistance against sintering as expressed by the equal $\mathrm{Pt}-\mathrm{Pt}$ coordination number after both LTR and HTR is probably due to prereduction of the catalysts. After HTR the Pt-Pt distance is shortened by $0.04 \AA$ with a noticeable increase in the Debye-Waller factor. This can be caused by a changing interaction with the support. From a combination of TPD and EXAFS experiments on platinum particles, which were highly dispersed into LTL zeolite $[7,8]$ it had been concluded that hydrogen is releasing from the metal-support interface during reduction at high temperatures. The long metal-oxygen distance of typically $2.7 \AA$ detected for the Pt/LTL catalyst after LTR was interpreted to be the result of the presence of hydrogen in the metal-support interface. In this study a metal-oxygen distance of $2.68 \AA$ is observed after LTR fully in agreement with the results for platinum particles dispersed in zeolite LTL and other metal particles (Ir, Rh) supported on $\gamma-\mathrm{Al}_{2} \mathrm{O}_{3}, \mathrm{TiO}_{2}$ and $\mathrm{MgO}$ (for a literature survey see Ref. [11]). The short metal-oxygen distance of $2.28 \AA$ observed here for $\mathrm{Pt} / \gamma-\mathrm{Al}_{2} \mathrm{O}_{3}$ after high temperature reduction was also found for Pt/LTL. Similar metal-oxygen distances were observed by EXAFS on reduced $\mathrm{Ir} / \gamma-\mathrm{Al}_{2} \mathrm{O}_{3}$ after evacuation and after oxygen 
Table 1

Results of refinement of structural parameters for $\mathrm{Pt} / \gamma-\mathrm{Al}_{2} \mathrm{O}_{3}$

\begin{tabular}{lllllr}
\hline Treatment & Backscatterer & $N$ & $R(\AA)$ & $\Delta \sigma^{2}\left(\times 10^{-3} \AA^{-2}\right)$ & $\Delta E_{0}$ \\
\hline R300 & Pt & $5.0(0.1)$ & $2.76(0.01)$ & $4.0(0.1)$ & $1.6(0.2)$ \\
R450 & $\mathrm{O}$ & $1.2(0.1)$ & $2.68(0.06)$ & $7.4(1.2)$ & $6.6(0.5)$ \\
& $\mathrm{Pt}$ & $5.0(0.2)$ & $2.72(0.01)$ & $4.9(0.3)$ & $5.0(0.6)$ \\
\hline
\end{tabular}
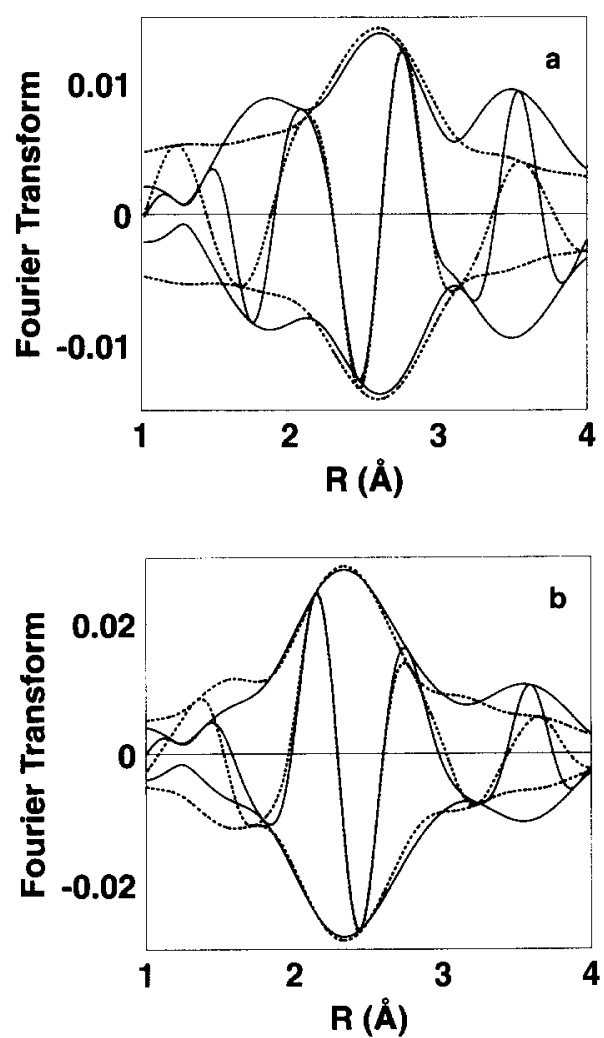

Fig. 4. Fourier Transform $\left(k^{1}, \Delta k=3.5-9.0 \AA^{-1}, \mathrm{Pt}-\mathrm{O}\right.$ phase corrected) of difference file (isolated EXAFS minus calculated $\mathrm{Pt}-\mathrm{Pt}$ contribution) (solid line) and calculated $\mathrm{Pt}-\mathrm{O}$ contribution (dotted line) for sample R300 (a) and sample R450 (b).

adsorption at $77 \mathrm{~K}[12]$. This distance is characteristic of oxygen atoms in direct contact with low- or zero-valent metal clusters [11].

It has to be stressed that the catalysts used in this study and in Refs. [7, 8] are not calcined in air before reduction. It is possible that calcination before reduction, which was done in other studies [13-15] will lead to a different structure in the metal support interface. More research is needed to investigate this.
Release of hydrogen from the metal-support interface with increasing reduction temperature alters the electronic properties of the platinum particles. A white line study of $\mathrm{Pt} / \gamma-\mathrm{Al}_{2} \mathrm{O}_{3}$ [16] shows that after LTR the platinum particles are electron rich $(8.6 \%$ less holes in the d-band than Pt bulk metal). HTR leads to slightly electron deficient $\mathrm{Pt}$ (0.6\% more holes than Pt bulk metal). We tend to believe that this change in electronic properties as a function of the reduction temperature causes the reported decrease in hydrogenolysis activity [1-6].

\section{References}

[1] G.J. den Otter and F.M. Dautzenberg, J. Catal. 53 (1978) 116.

[2] P.G. Menon and G.F. Froment, Appl. Catal. 1 (1981) 31

[3] R. Kramer and M. Andre, J. Catal. 58 (1979) 287.

[4] J. Margitfalvi, P. Szedlacsek, M. Hegedüs and F. Nagy, Appl. Catal. 15 (1985) 69.

[5] R. Kramer and M. Fischbacher, J. Mol. Catal. 51 (1989) 247.

[6] S.I. Abasov, V.Y. Borovkov and V.B. Kazansky, Catal. Lett. 15 (1992) 269.

[7] J.T. Miller, B.L. Meyers, G.S. Lane, M. Vaarkamp and D.C. Koningsberger, J. Catal. 143 (1993) 395.

[8] M. Vaarkamp, F.S. Modica, J.T. Miller and D.C. Koningsberger, J. Catal. 144 (1993) 611.

[9] D.C. Koningsberger, in: Hercules Vol. II, eds. J. Baruchel, J.L. Hodeau, M.S. Lehmann, J.R. Regnard and C. Schlenker (Springer, Berlin, 1993) p. 213.

[10] F.W. Lytle, D.E. Sayers and E.A. Stern, Physica B 158 (1988) 701

[11] D.C. Koningsberger and B.C. Gates, Catal. Lett. 15 (1992) 271.

[12] F.W. Kampers and D.C. Koningsberger, Faraday Discuss. Chem. Soc. 89 (1990) 137.

[13] P. Lagarde, T. Murata, G. Vlaic, E. Freund. H. Dexpert and J.B. Bournonville, J. Catal. 84 (1983) 333.

[14] F.W. Lytle, R.B. Greegor, E.C. Marques, D.R. Sandstrom, G.H. Via and J.H. Sinfelt, J. Catal. 95 (1985) 546.

[15] E.S. Shpiro, R.W. Joyner, K.M. Minachev and P.D.A. Pudney, J. Catal. 127 (1991) 336.

[16] M. Vaarkamp, J.T. Miller, F.S. Modica, A.N. Mansour and D.C. Koningsberger, J. Phys. Chem., submitted. 